\title{
$\mathfrak{3} \mathfrak{n} \mathfrak{h} \mathfrak{a} \mathfrak{l} \mathfrak{t}$.
}

\section{Erfter gliets.}

\section{gaienflitider Herterridit.}

1. Die Pfltijten. Seite.

1. Berpflidtung zum Dienite . . . . . . . . . . 1

2. Pflidten bee Soldaten . . . . . . . . . . . 1

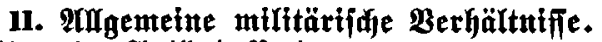

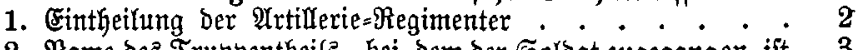

2. Rame Des Truppentheils, bei bem ber Soldat zugegangett ift 3

3. Ramen ber Borgejesten . . . . . . . . . . 3

4. Rangoronutg unb Srabauzzeifintug . . . . . . . 3

11I. Berhältnif bes Solbaten.

1. Die Borgeję̧ten

A. $8 \mathfrak{u}$ ben $\mathfrak{B}$ orgejegten

2. Suborbination . . 6

3. Ghrenbezeifinung . . . . . . . . . . . . . 6

4. Bertrauten zul Den Borgejeşten . . . . . . . . 8

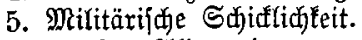

a) $\mathfrak{s} \mathfrak{m} \mathfrak{e r g}$ gemeinent.

b) Beim Buruf

c) Beint Reben . . . . . . . . . . . . . . 9

d) Bei Beffeibung . . . . . . . . . . . . . . 9

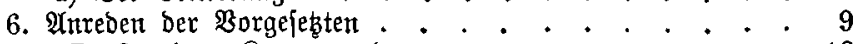

B. $8 \mathfrak{H}$ ben $\mathfrak{A} \mathfrak{a} \mathfrak{e} \mathfrak{r} \mathfrak{a} \mathfrak{e} \mathfrak{n} . \quad . \quad . \quad . \quad . \quad . \quad .10$

C. Bu Berjonen anderer Stände. . . . . 10

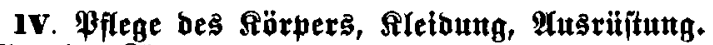

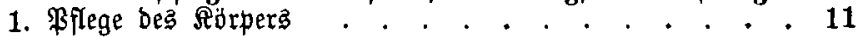

2. Rleibung.

a) IUgemeines . . . . . . . . . . . . . . . 12

b) Reinigung ber einzelnten Stüđe . . . . . . . 13

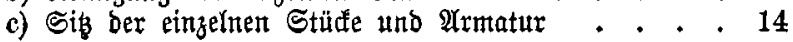




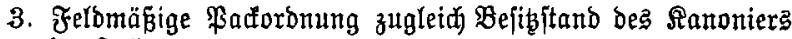
im Frelbe.

a) Tornifter

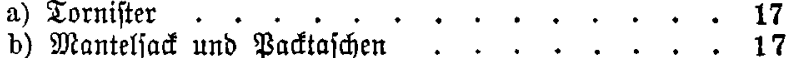

c) Brodjaff . . . . . . . . . . . . . 18

4. Felbmäßjige Ylužlegoronung . . . . . . . . . . 19

\section{v. Berlyalten bes Solbaten in Dienite.}

1. Aflfgemeines

2. Эัm Bried

3. Bei Befehlen

4. Bei Melbungen

5. Bei Bitten

6. Bei Beidmerden.

a) Ueberlegung . . . . . . . . . . . . . 23

b) $\mathfrak{A}$ nbringung . . . . . . . . . . . . . 23

c) Beitere Berwentung . . . . . . . . . . . . 24

v1. Berfyalten bes Solbaten anjer Dienit . . . 24 V11. DrbnunB in ber Maferne.

1. AIgemetne Boridriften . . . . . . . . . . . 26

2. In ben 3 immern . . . . . . . . . . . . . 26

3. Berhalten in Den Binmern . . . . . . . . . . 27

4. Berfalten bcim Menagiren . . . . . . . . . . 30

5. Rufje bci Tage . . . . . . . . . . . . . 30

6. Benehmen in ben Bimmern . . . . . . . . . . 30

7. Seim Ricberlegen. . . . . . . . . . . . . 30

8. $\mathfrak{A}$ \legoronutg in ben Bimmern bet ötonom. Bijptationer 31

9. Bimmerbienit . . . . . . . . . . . . . . 34

10. Rodtour und Menageoronung . . . . . . . . . 35

\section{V11. Dronung int Stall.}

1. OUgemeine Bejtimmung . . . . . . . . . . . 35

2. Futter . . . . . . . . . . . . . . 36

3. Borjdrijten beim f̧üttern . . . . . . . . . . 36

4. Tränter . . . . . . . . . • • . • • • • 37

5. Etrelt . . . . . . . . . . . . . . . 37

6. Etallzeiten . . . . . . . . . . . . . . 37

a) §rüh = und Mittagfutter . . . . . . . . . 38

b) ßuken . . . . . . . . . . 38

c) AGendfutter . . . . . . . . . . . . 39

d) Rađtränten . . . . . . . . . . . . . 39

7. Wartung ber yom Dienit eingerüđten ßferbe . . . . 39

8. Wehandiung Der Pferde . . . . . . . . . . . 40

9. Stalltadje . . . . . . . . . . . . . 40

10. Bei b̈lonomijüen Bifitationen . . . . . . . . . 41 
11 Bferdevifitationen

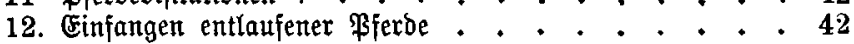

13. Sdjemmen ber \$ferde. . . . . . . . . . . 42

\section{1x. Gebihnen.}

1. Эnt ber Sarnifon

2. Эm Lager Lechfeld . . . . . . . . . . . . . 45

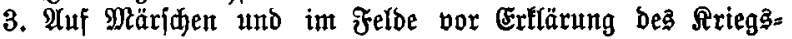
zuttandes. . . . . . . . . . . . . . 45

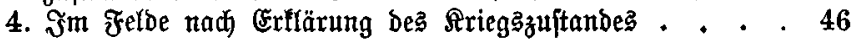

5. \$reife und Tragzeit ber Monturf́tüfe . . . . . . . 46

1. Drben und Medaillen . Pelohaungen.

2. Belobung . . . . . . . . . . . . . . . 48

3. Denfzeiden . . . . . . . . . . . . . . 48

4. Dienitalterszeiden . . . . . . . . . . . . . 48

xl. Beftrafungett.

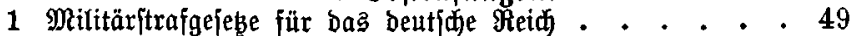

2. Disciplinaritrafen . . . . . . . . . . . . . 51

3. Egrenftrafen . . . . . . . . . . . . . . 51

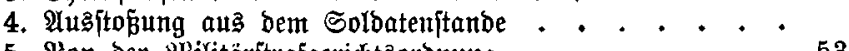

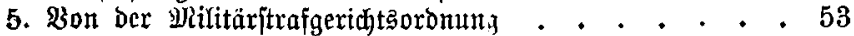

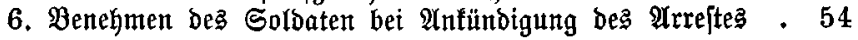

X11. $\mathfrak{u}$ rlautb . . . . . . . . . . . 54

X11. Grfranfung . . . . . . . . . . . . 56

Xiv, Berforgung invaliber Solbaten, Huteritïls= ung ber sinterbliebenen, beren bebiurftigen Angehörigen ber Oicferviftent und Qand. wehrmäntrer ............ 57

XV. Garnijonabienit.

1 Madtotenjt . . . . . . . . . . . . 60

2. Feuerpiquet . . . . . . . . . . . . . . 62

3. Drbonanzoienjt . . . . . . . . . . . . . 62

4. Sdubbadie ober Sauvegarbe . . . . . . . . . 63

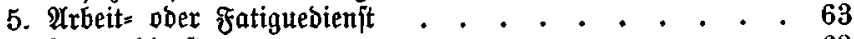

6. Fugrendienft . . . . . . . . . . . . . . . 63

1. Borbereitung . . . . . 64

2. Mährend des Marjđes . . . . . . . . . . . 64

3. Berhalten in Duartiere . . . . . . . . . . . 66

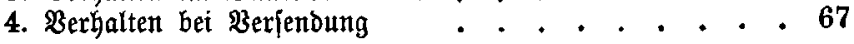

XV11. Bon ber Mebrverfafiung . . . . . . . 68 
XV111 Die Armee.

Seite.

1. Reptandtheile ber Armee, ihre Uniformirung und Betwaffnung 69

2. Armee=Eintheilung und Dialocirung im Frieben . . . 71

3. Artilferie . . . . . . . . . . . . . 75

4 Armee=Eintheilung im Ŝriege . . . . . . . . . 75

\section{Bweiter gheif.}

\section{Artillerifti[der Enterridt.}

1. Beftattotheile . . . . . . . . . . 77

\section{Daş $\mathfrak{B u l}_{\mathfrak{u} \text { toer. }}$}

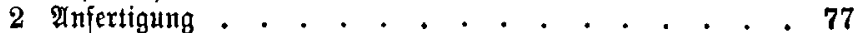

3. Pulverjorten . . . . . . . . . . . . . , . 78

4. Rennzeiden des \$ulvers.

a) Des guten $\mathfrak{P u l v e r s}$. . . . . . . . . . . 78

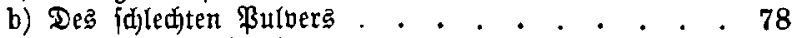

c) Urjađien und atbilfe . . . . . . . . . . 79

d) Seritellung juabjafter Patronen . . . . . . . 79

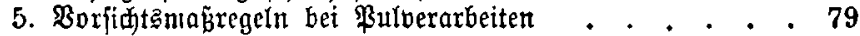

a) $\mathscr{A}$ Igemeines Berthalten . . . . . . . . . . 80

b) Berfalten in Mayazinen . . . . . . . . . 80

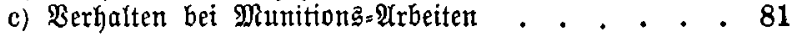

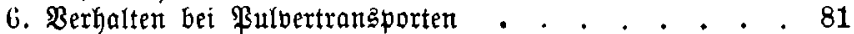

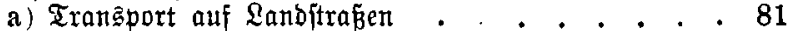

b) Tramaport auf Eifenbaljnen . . . . , . . 84

c) Transport zu खaffer . . . . . . . . . . . . 84

11. Gejüübe.

1. Einleitung . . . . . . . . . . . . , 85

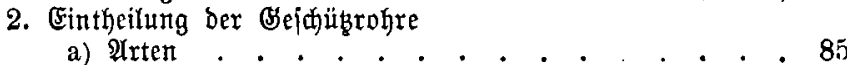

b) アaliber . . . . . . . . . . . . . . 85

c) Bebraudgatafien . . . . . . . . . . . 86

3. Gejchükrohre . . . . . . . . . . . . 86

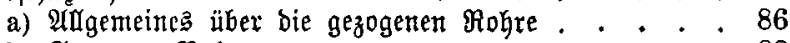

b) Bezogente Rolire . . . . . . . . 88

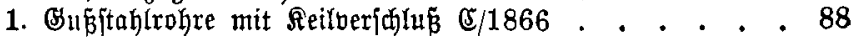

2. Bronzrohre mit Reilveridlus $\mathbb{E} / 1<66$. . . . . . . 91

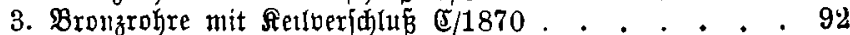

4. $9 \mathrm{~cm}$. Stahlfanone mit Ћolbenverichlǘ . . , . . 93

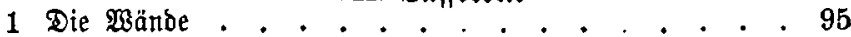

2. Die ßiegel . . . . . . . . . . . . . . . 96

3. Die Ridntmafhine . . . . . . . . . . . . 96 
4. Die Yre . . . . . . . . . . . . . . . . 97

5. Die Räber . . . . . . . . . . . . . . . 97

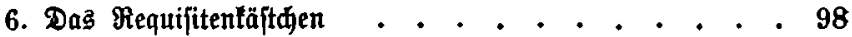

\section{1v. Dite Proket.}

1. Das Untergeftell . . . . . . . . . . . . . 98

2. Der Rafter . . . . . . . . . . . . . . 99

v. Die Magen.

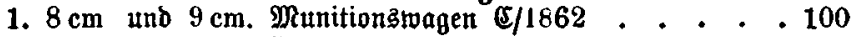

2. Munitionstagen $\mathbb{E} / 1836$. . . . . . . . . . . 101

3. Aptixter Infanterie-Munitionstwagen $\mathbb{E} / 1872$. . . . 102

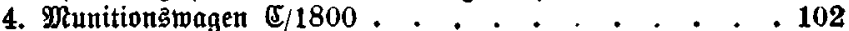

5. Jelbfdmiebe $\mathbb{E} / 1836$. . . . . . . . . . . . 102

6. Batteriewagen . . . . . . . . . . . . . 103

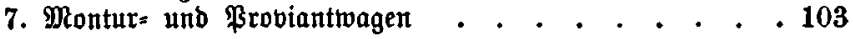

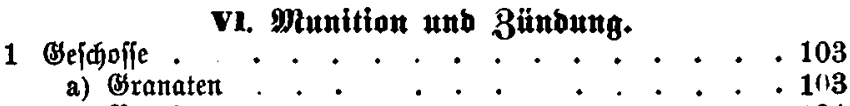

a) Brandgranaten $. . \quad . \quad . \quad . \quad . \quad . \quad . \quad 104$

c) Unterfaceibung ber blinblaborirten bon ben fđarflabo= ritten Befdopent . . . . . . . . . 105

d) Büđjenfartätłd)en . . . . . . . . . 106

2. Batronen . . . . . . . . . . . . . . . 1116

3. Bündungen . . . . . . . . . . . . . . . 107

4. Behandlung der Munition . . . . . . . . . 107

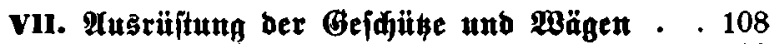

1. Die $9 \mathrm{~cm}$. Brej巾üb=ßrobe . . . . . . . . . . 109

2. Das $9 \mathrm{~cm}$. Rohr und bie Laffete. . . , . . . . 111

3. Die $9 \mathrm{~cm}$. Bager=ßroge . . . . . . . . . . 111

4. $9 \mathrm{~cm}$ Muntion 3 -Sintertwagen $\mathbb{5} / 1862 \quad$. . . 112

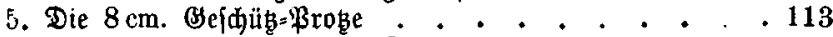

6. Das $8 \mathrm{~cm}$ ßohr und bie Laffete. . . . . . . . 114

7 Die $8 \mathrm{~cm}$. Magent=ßrobe . . . . . . . . . . 114

8 Der $8 \mathrm{~cm}$. Munitions=5interwagen . . . . . . 115

9. Feld fdmiebe

10. Der 1. Batteriemagen als Requifitentwagen . . . . 116

11. Der 2 Batterietragen . . . . . . . . . . . 116

12. Montur $=$ und Provtant $=\mathfrak{W a g e n} \quad . \quad . \quad . \quad . \quad . \quad . \quad . \quad 117$

14. Berpafung bei ben Eolonen . . . . . . . . . 117

v11. Bebienumb ber Gejuithe.

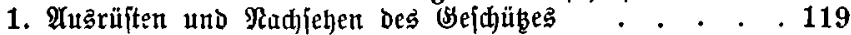

2 Bebrenen des Ge\{dü̈ве . . . . . . . . . . 122

1X. Sdjefenen unb Werfen. 


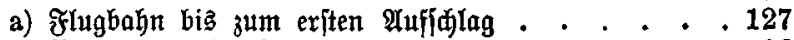

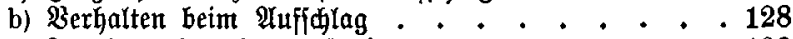

c) Flugbahn ber Sprengftüfe . . . . . . . . 129

2. Trefffähigleit ber Bejchübe . . . . . . . . . . 129

3. Wirfung bez Bejđoßßez auf bas Biel . . . . . . . 130

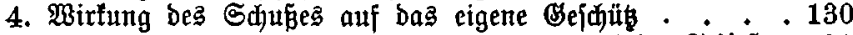

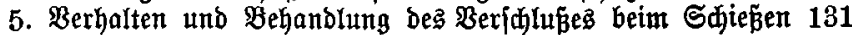

6. Der Jebrau出 des Branat\{dujez . . . . . . . . 133

7. Der Brandgranatidu

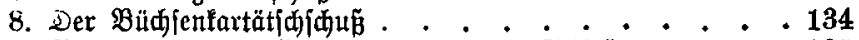

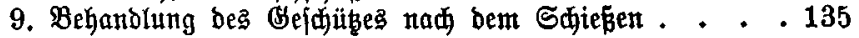

X. Sanobabung ber Geidühe unt Jahrzenge in

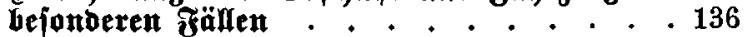

1. Wedjeln eines $\mathfrak{Q}$ affetenrades. . . . . . . . . . 136

2. Bedfeln eines Sinterrades bei ben andern Fahrzengen . 137

3. Ablegen Des ßohrez . . . . . . . . . . . 137

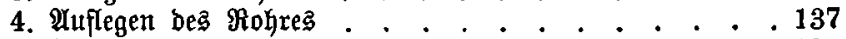

5. Berfühten bes Mohrez unter der \$roge . . . . . . 137

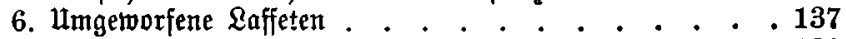

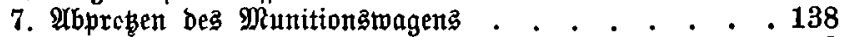

8. Bewegung abgeprobzter $9 \mathrm{~cm}$. Beffübęe mit 8 uggurten .138

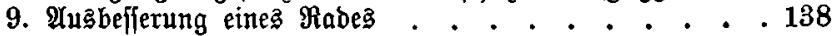

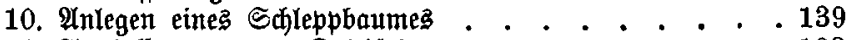

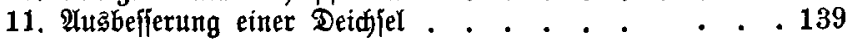

x1. Batteriebaut.

1. Anfertigung ber Materialien zur Betleibung . . . . 139

2. Serftellung Der Bef̌leibungen . . . . . . . . . 142

3. Einridfung einer Felojhanze und ber zugehörigen 2 Anlagen

im erugemeinen............ .143

4. Berhalten beim Batteriebau . . . . . . . . . 144

x11. Die Pifitole.

1. Bejureibung . . . . . . . . . . . . . . . 144

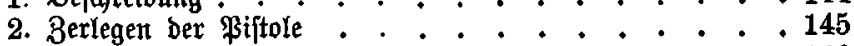

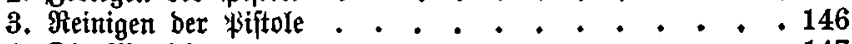

4. Die Munition . . . . . . . . . . . . 147

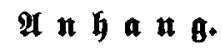

Cintheilung und Dislotation ber Artillerie. 